\title{
Empleo del vidrio volcánico en el hormigón celular
}

\author{
The use of vulcanic glasses in cellular concrete
}

fecha de recepción: 18-VI-96

feccha de aceptación:4-DX-96

\section{RESUMEN}

Se hace un estudio con diferentes mezclas, teniendo como variables la arena silice, el vidrio volcánico y el polvo de aluminio.

Al hormigón celular, una vez curado en autoclave, se le hace un estudio de resistencia a la compresión, densidad en estado seco y difracción de rayos $X$, llegándose a la conclusión de que los mjores resultados se obtienen cuando se emplea el vidrio volcánico al $50 \%$ en sustitución de la arena sílice.

Cuando se disminuye el porcentaje de polvo de aluminio de $0,11 \%$ a $0,09 \%$, se presenta un ligero aumento en la densidad, con un notable crecimiento de la resistencia a la compresión.

\author{
J. L. ALVAREZ, División de Materiales. CTDMC, La Habana; \\ N. VEGA, LACEMI. "J. I. DEL CORRAL", La Habana
}

\section{INTRODUCCIÓN}

Los hormigones celulares están formados por aglomerantes, los cuales pueden ser cemento, cal o ambos, más un agregado que fundamentalmente es arena sílice; aunque la literatura habla de tobas volcánicas y colas de caolín $(1,2,3,4,5,6)$, aditivos, agua $\mathrm{y}$ un producto formador de espuma o poros, que, en dependencia del empleado, da lugar a un hormigón gaseoso o espumoso.

\begin{abstract}
SUMMARY
This paper covers studies made using different mixes for cellular concrete variating silica sand, vulcanic glass and aluminium powder contents.

Studies on compressive strenght, dry density and X-ray diffraction are performed on autoclave cured cellular concrete, concluding that best results are attained when vulcanic glass contents reaches $50 \%$ of silica sand.
\end{abstract}

When percentage of aluminium powder is diminished from $0.11 \%$ to $0.09 \%$, alight increase in density, with noticiable growth of comprensive strength are observed. 
En este caso se trabaja con polvo de aluminio, obteniéndose un hormigón del tipo gaseoso.

El objetivo de este trabajo es emplear vidrio volcánico en sustitución de la arena sílice en la producción del hormigón celular tipo gaseoso, disminuyendo también el contenido de aluminio, obteniendo un producto ligero y resistente.

\section{MATERIALES Y MÉTODOS}

En el desarrollo del trabajo, se emplearon los siguientes materiales:

- Cemento Portland P-350

- Arena sílice

- Vidrio volcánico

- Hidrato de cal (calidad II)

- Yeso de fraguado lento

- Polvo de aluminio

- Hidróxido de sodio (48\%)

- Agua potable

El esquema de trabajo fue el siguiente:

1. Análisis químico de las materias primas utilizadas.

2. Preparación de las dosificaciones.

3. Obtención de el hormigón

4. Medición de las propiedades físicas y análisis de la significación.

5. Determinación de las fases que se forman.

La difracción de rayos $\mathrm{X}$ se realizó a las muestras en un difractómetro Phillips PW 1750, luego de pulverizadas hasta $0,1 \mathrm{~mm}$ en morteros de ágata.

\section{RESULTADOS Y DISCUSIÓN}

El análisis químico de los materiales utilizados se presenta en la Tabla I.

Se realizaron dosificaciones, variando el \% de arena sílice y aluminio en 100,75 , y $50 \%$, la arena vs, vidrio $\mathrm{y}$ el aluminio en 0,11 y $0,09 \%$.

A todos los hormigones obtenidos con estas mezclas se les practicaron los ensayos de densidad $\sigma$, resistencia a compresión $\mathrm{R}$, crecimiento al borde del molde $\mathrm{y}$ temperatura de fundición, tal y como se presentan en la Tabla II.

Para la confección de los morteros se emplearon moldes cúbicos de $100 \times 100 \times 100 \mathrm{~mm}$, los cuales fueron calentados a $60^{\circ} \mathrm{C}$ y engrasados antes del vertido del
In our study, the use of aluminium powder as expansive, gives a gassy concrete.

The main scope of this study is to use vulcanic glass instead of silica sand in order to produce a gassy type cellular concrete, diminishing also aluminium contents, and so to attain a lightweight and resistant product.

\section{MATERIALS AND METHODS}

During this study, this materials where used:

- Portland cement P-350 type

- Silica sand

- Vulcanic glass

- Hydrated lime (II quality)

- Low-setting gypsum

- Aluminium powder

- Sodium hydroxide (48\%)

- Potable water.

Working path was as follows:

1. Chemical analysis of materials used.

2. Mix design.

3. Making of concrete.

4. Measurement of physical properties of concrete, analysis of significance.

5. Determination of phases formed.

$X$-ray diffraction was made to samples in a Phillips $P W 1750$ diffractomer after grinding down to $0,1 \mathrm{~mm}$ size in an agat mortars.

\section{RESULTS AND DISCUSSION}

Table I shows chemical analysis of used materials.

Mixes were made varying \% of silica sand and aluminium powder in 100, 75 and 50\%, sand to glass and aluminium from 0.11 and 0,09\%.

Tests of density, $\sigma$, compressive strenght, $R$, overgrowth from mould edge and setting temperature, as shown in Table II, were performed over all samples of concrete prepared with designed mixes.

For casting all concretes, cubic moulds $100 \times 100 \times$ $100 \mathrm{~mm}$ were used, after a previous heating up to $60^{\circ} \mathrm{C}$ and a light grease covering of sides and bottom. 
TABLA I (TABLE I)

Análisis químico de las materias primas (Chemical analysis of raw materials)

\begin{tabular}{|c|c|c|c|c|c|c|}
\hline & UM & $\begin{array}{c}\text { Cemento } \\
\text { (Cement) } \\
\text { P-350 }\end{array}$ & $\begin{array}{l}\text { Arena Silice } \\
\text { (Silica Sand) }\end{array}$ & $\begin{array}{c}\text { Hidrato de } \\
\text { Cal } \\
\text { (Hydrated } \\
\text { Lime) }\end{array}$ & $\begin{array}{c}\text { Vidrio Volc. } \\
\text { (Vulcan. } \\
\text { Glass) }\end{array}$ & $\begin{array}{c}\text { Yeso Lento } \\
\text { (Gypsum) }\end{array}$ \\
\hline $\mathrm{SiO}_{2}$ & $\%$ & 22,10 & 98,84 & -- & 68,10 & --- \\
\hline $\mathrm{Al}_{2} \mathrm{O}_{3}$ & $\%$ & 4,72 & 0,39 & $\cdots$ & 11,78 & -- \\
\hline $\mathrm{Fe}_{2} \mathrm{O}_{3}$ & $\%$ & 3,40 & 0,27 & -- & 1,79 & -- \\
\hline $\mathrm{CaO}$ & $\%$ & 62,24 & -- & 84,74 & 1,84 & 35,74 \\
\hline $\mathrm{SO}_{3}$ & $\%$ & 2,44 & -.. & 0,31 & 0,30 & 45,24 \\
\hline PPI & $\%$ & 2,45 & 0,32 & 26,41 & 7,80 & -- \\
\hline RI & $\%$ & 2,05 & 0,18 & 20,76 & --- & --. \\
\hline
\end{tabular}

hormigón. El hormigón fue preparado en una mezcladora, empleando agua caliente, con el objetivo de que el producto final alcanzara una temperatura de, aproximadamente $46^{\circ} \mathrm{C}$.

Todos los materiales se vertieron en la mezcladora, excepto el polvo de aluminio, controlándose el tiempo de mezclado del cemento y del aluminio. Una vez que el hormigón está dentro del molde se determinó la temperatura y crecimiento al borde del molde. El hormigón celular fue desmoldado a las $24 \mathrm{~h}$ y puesto en autoclave por espacio de $10 \mathrm{~h}$ de acuerdo con el siguiente ciclo de curado: aumento de temperatura y presión por 2 $\mathrm{h}$, curado a presión constante de $12 \mathrm{~kg} / \mathrm{cm}^{2}$ durante $6 \mathrm{~h}$ y descenso de temperatura y presión por $2 \mathrm{~h}$.

La medición de la resistencia a compresión y de la densidad se realizó por triplicado, para realizar el análisis de varianza de estos parámetros, con la variación del \% de vidrio volcánico y de polvo de aluminio, realizándose un total de 24 mediciones.

Obteniéndose para la $\sigma$ :

$\mathrm{F}_{\mathrm{A} 1}=3,8$ mientras que la $\mathrm{F}(2,8)$ con $95 \%$ de significación es 4,46

y

$\mathrm{F}_{\mathrm{vv}}=24,4$ mientras que la $\mathrm{F}(4,8)$ con $95 \%$ de significación es 3,84

Para la resistencia $\mathrm{R}$ :

$\mathrm{F}_{\mathrm{A} 1}=13,57$ mientras que $\mathrm{F}(2,8)$ con $95 \%$ de significación es 4,46
Concrete mixes were made in a lab mixer using hot water, aiming for final product temperature about $46^{\circ} \mathrm{C}$.

All materials were put together into mixer's bowl except aluminium powder, controlling mixing time of cement and aluminium. Once poured the fresh mix of concrete inside forms, temperature and overgrowth from mould edge were measured. Cellular concrete was taken off forms after 24 hours and put to autoclave for a 10 hours cycle, following these steps: rise temperature and pressure, 2 hours, constant pressure of $12 \mathrm{~kg} / \mathrm{cm}^{2}, 6$ hours, and 2 hours for relief of pressure and temperature.

Testing for compressive strenght and density were performed three times each in order to determine variance analysis regarding per-cent variation of vulcanic glass and aluminium powder, giving 24 tests in the whole.

Results for $\sigma$ were:

$F_{A I}=3.8$ as $F(2.8)$ with $95 \%$ significance is 4.46

and

$F_{w}=24.4$ as $F(4.8)$ with $95 \%$ significance is 3.84

For strenght R:

$F_{A I}=13.57$ as $F(2.8)$ with $95 \%$ significance is 4.46 


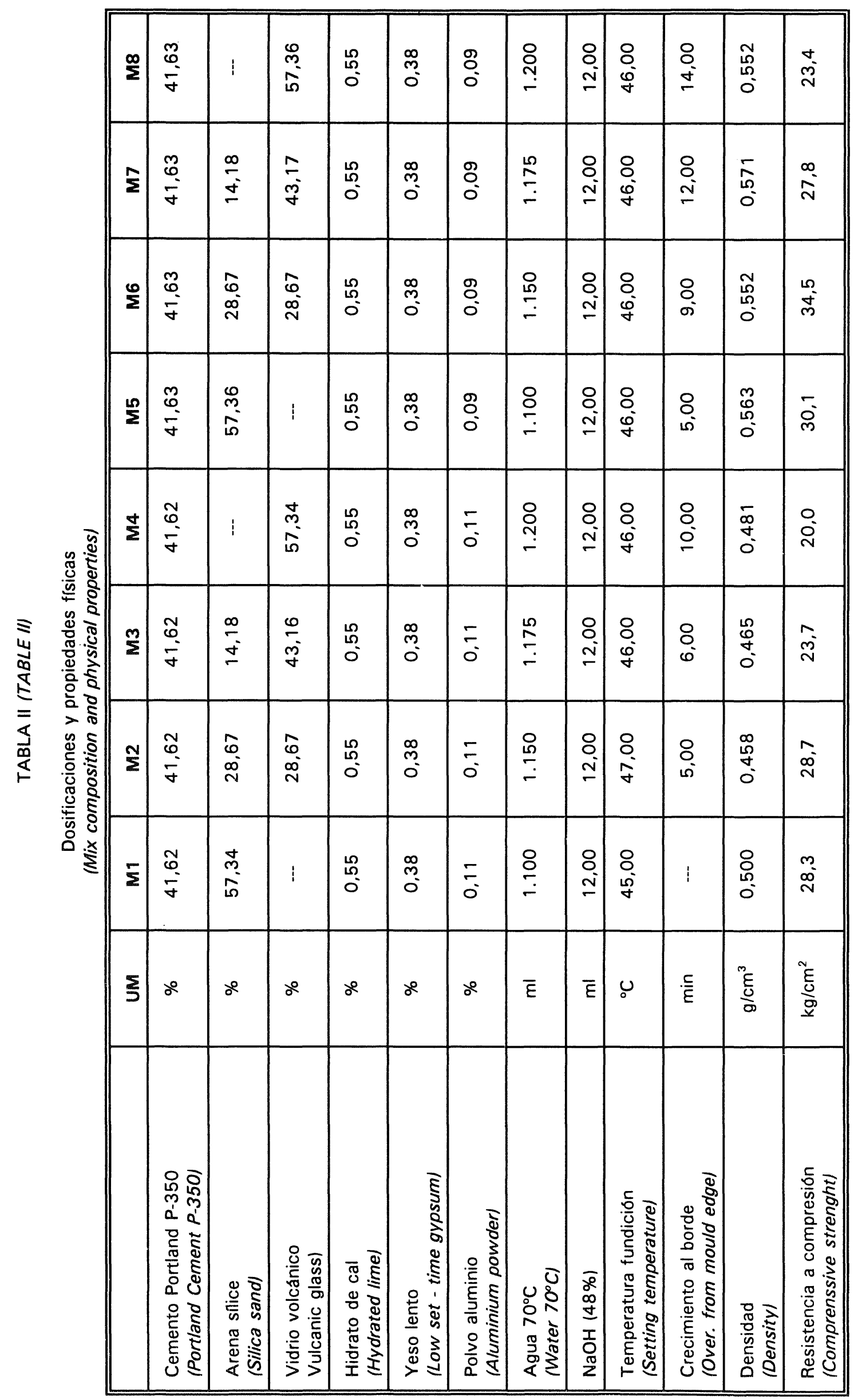


$\mathrm{F}_{\mathrm{vv}}=4,20$ mientras que $\mathrm{F}(4,8)$ con $95 \%$ de significación es 3,84 .

Los parámetros establecidos para la resistencia y la densidad son:

\section{$\mathrm{R}>25 \mathrm{~kg} / \mathrm{cm}^{2}$ y $\sigma=0,5 \mathrm{~g} / \mathrm{cm}^{2}$}

$\mathrm{El}$ análisis por difracción de rayos $\mathrm{X}$, evidenció, como fases cristalinas fundamentales, la tobermorita de 11 $\mathrm{A}^{\circ}$, el cuarzo y la calcita, en las proporciones que aparecen en la Tabla III. La formación de tobermorita de $11 \mathrm{~A}^{\circ}$ favorece la resistencia a la compresión.

En el proceso de conformación de la mezcla se observó que, a medida que aumenta el \% de vidrio volcánico, aumenta la cantidad de agua que hay que adicionar para alcanzar la fluidez requerida; ésta no varía al cambiar el \% de polvo de aluminio.

La temperatura de fundición se mantuvo prácticamente constante, lo cual se logró sin mayor dificultad.

El crecimiento al borde fue más lento con el aumento del vidrio volcánico, aunque la porosidad y homogeneidad del hormigón celular fue buena.

Las densidades se comportan normalmente, observándose que los mayores valores de resistencia a compresión se obtienen cuando se emplea el $50 \%$ de arena y $50 \%$ de vidrio volcánico, y es independiente del $\%$ de polvo de aluminio adicionado, tal y como se refleja en el análisis de la varianza.
$F_{\nu}=4.20$ as $F(4,8)$ with $95 \%$ significance is 3.48

Parameters stablished for strenght and density are:

$R>25 \mathrm{~kg} / \mathrm{cm}^{2}$ and $\sigma=0.5 \mathrm{~g} / \mathrm{cm}^{2}$

$X$-ray diffraction analysis showed as main crystalline phases, tobermorite $11 \mathrm{~A}^{\circ}$ quarts and calcite in proportions shown in Table III. Forming at $11 \mathrm{~A}^{\circ}$ tobermorite favoreus compressive strenght.

During mix forming process, it was noted that as the percent of vulcanic glass increases, a certain amount of water has to be added in order to attain required fluidity, as amount of water does not change while changing amount of aluminium powder.

Setting temperature was kept practically constant, without troubles.

Overgrowth over form lid top was slower respecting increase of vulcanic glass, even when porosity and homogeneity of cellular concrete were good.

Behaviour of densities were considered normally, observing higher values of compressive strenght were obteined when $50 \%$ of sand and 50\% of vulcanic glass were used, non depending upon \% aluminium powder added, as it's shown in variance analysis.

TABLA III (TABLE III)

Fases Cristalinas Fundamentales en el Homigón Celular

(Fundamental crystalline phases in cellular concrete)

\begin{tabular}{||l|c|c|c|}
\hline \multicolumn{1}{|c|}{$\begin{array}{c}\text { Muestras } \\
\text { (Samples) }\end{array}$} & $\begin{array}{c}\% \text { Tobermorita } \\
\text { (\% Tobermorite) }\end{array}$ & $\begin{array}{c}\% \text { Calcita } \\
\text { (Calcite) }\end{array}$ & $\begin{array}{c}\% \text { Cuarzo } \\
\text { (\% Quartz) }\end{array}$ \\
\hline Patrón W \% Al & 12 & 0,33 & 41 \\
\hline $50 \%$ V.V. 11\% Al & 77 & 0,30 & 18 \\
\hline $75 \%$ W 11\% Al & 58 & 0,49 & 8,75 \\
\hline $100 \%$ V.V. 11\% Al & 72 & 0,27 & 1 \\
\hline Patrón 9 \% Al & 22 & 0,46 & 41 \\
\hline $50 \%$ W 9 $\%$ Al & 67 & 0,42 & 8 \\
\hline $75 \%$ V.V. 9 \% Al & 59 & 0,37 & $\cdots$ \\
\hline $100 \%$ V.V. 9 \% Al & 57 & 4,10 & 18 \\
\hline
\end{tabular}




\section{CONCLUSIONES}

1. Sustituyendo el $50 \%$ de la arena por vidrio volcánico se logra obtener un hormigón celular con mayor resistencia a compresión y una ligera disminución de su densidad, por lo que se obtiene un producto final con mejores características que el empleado tradicionalmente.

2. La tobermorita formada con estas formulaciones es la de $11 \mathrm{~A}^{\circ}$, con una relación de 0,75 de intensidad entre las reflexiones $2,95 \mathrm{~A}^{\circ} / 11 \mathrm{~A}^{\circ}$.

3. La mayor cantidad de tobermorita se forma con la dosificación de $50 \%$ de vidrio y $50 \%$ de arena de sílice.

\section{CONCLUSIONS}

1. Sustituting $50 \%$ of sand for vulcanic glass, it's possible to obtain a cellular concrete with higher compressive strenght and a light density decrease, which means a best featured product than formely employed.

2. Tobermorite attained with these mixes is of $11 A^{\circ}$, with a 0.75 relation of intensity between reflexions of $2.95 \mathrm{~A}^{\circ} / 11 \mathrm{~A}^{\circ}$.

3. Higher amount of tobermorite is formed with a $50 \%$ of glass and $50 \%$ silica sand.

\section{BIBLIOGRAFÍA}

(1) ÁLVAREZ, J.L. y RIVERA, N.: (1984). “Influencia de diferentes tobas cubanas en la producción del hormigón celular". Ponencia. IV Conferencia Científica ISPJAE. Habana.

(2) GÓMEZ, M y ÁLVAREZ, J.L.: (1984). "Estudio e escala experimental de la influencia de la granulometría de las zeolitas en algunos parámetros del hormigón celular". Ponencia. IV Conferencia Científica ISPJAE. Habana.

(3) ESCALONA, I., GÓMEZ, M. y ÁLVAREZ, J.L.: (1987). “Composición del hormigón ligero y procedimiento de obtención". Patente No. 21759. Habana.

(4) GÓMEZ, M., ÁLVAREZ, J.L. y RIVERA, N.: (1985). "Hormigón celular sobre la base de tobas". Informe final. Tema 046.18. Habana.

(5) RIVERA, N., GÓMEZ, M. y ÁlVAREZ, J.L.: (1984). “Aprovechamiento de las colas de caolín en la producción de hormigón celular". IV Concurso BTJ. Habana.

(6) RIVERA, N., GÓMEZ, M. y ÁLVAREZ, J.L.: (1989). “Composición para la producción de hormigón celular a partir de las colas de caolín". Patente 21850. Habana. 